Numer i cal anal ysi s of the noi se reduction ef fect by super positi on of hi gh frequency cur rent in sem conduct or I aser s

\begin{tabular}{|l|l|}
\hline 著者 & I m an A. Sazzad MS. , Yamada M nor u \\
\hline $\begin{array}{l}\text { j our nal or } \\
\text { publ i cat i on t i t l e }\end{array}$ & $\begin{array}{l}\text { Paci f i c Ri m Conf er ence on Laser s and } \\
\text { El ect r o- Opt i cs, CLEO - Techni cal Di gest }\end{array}$ \\
\hline number & 2013 \\
\hline page range & 6600427 \\
\hline year & 2013 01- 01 \\
\hline URL & ht t p: //hdl . handl e. net /2297/36266 \\
\hline
\end{tabular}




\title{
Numerical Analysis of the Noise Reduction Effect by Superposition of High Frequency Current in Semiconductor Lasers
}

\author{
A. Sazzad M.S. Imran and B. Minoru Yamada \\ Division of Electrical and Computer Engineering, Graduate School of Natural Science and Technology \\ Kanazawa University, Kakuma-machi, Kanazawa, Ishikawa, 920-1192, Japan
}

\begin{abstract}
Suppression phenomena of optical feedback noise in semiconductor lasers by the superposition of high frequency current have been numerically analyzed and explained with approximated but analytical equations. Correspondence between experiment and simulation is also demonstrated.
\end{abstract}

\section{INTRODUCTION}

Semiconductor lasers play a central role in the growing world of optoelectronic technologies. Optical feedback (OFB) is induced by the re-injection of output light into the laser followed by reflection at the surface of connecting optical devices and causes excess noise in the output of the laser [1]. This OFB induced excess noise degrades the performance of the system.

The OFB noise is well suppressed by superposition of high frequency (HF) current if frequency and amplitude of the superposed current are suitably selected. However, it has been experimentally reported that the OFB noise is not suppressed when frequency of the superposed current and round-trip frequency of the OFB are in relation of rational numbers [2]. A theoretical analysis based on mode competition phenomena and external cavity modes was also given in [2]. But quantitative assessment for conditions unable to suppress OFB noise was difficult from the small signal analysis.

In this paper, we present a new improved model by which generation of the OFB and its suppression by the superposition of HF current can be numerically simulated. Conditions unable to suppress the OFB noise are evidently shown. The model used here is based on multimode rate equations that include non-linear gain, Langevin noise sources, OFB and HF superposition. Our model is applied to $850 \mathrm{~nm}$ GaAs lasers.

\section{Model of Analysis}

Operation of a semiconductor laser under OFB is illustrated in Fig. 1, where output light from the laser is reflected back from an external mirror and re-injected into the laser.



Fig. 1: Operation of a semiconductor laser under optical feedback.

Mode dynamics of semiconductor lasers operating under external OFB are governed by the following rate equations of the modal photon number $S_{p}(t)$, modal phase $\theta_{p}(t)$ and number of injected electrons $N(t)$ [3].

$$
\begin{aligned}
\frac{d S_{p}}{d t} & =\left(G_{p}-G_{\text {tho }}+\frac{c}{n_{r} L} \ln \left|U_{p}\right|\right) S_{p}+\frac{a \xi N / V}{\left[2 \frac{\left(\lambda_{p}-\lambda_{0}\right)}{\delta \lambda}\right]^{2}+1}+F_{S p}(t) \\
\frac{d \theta_{p}}{d t} & =\frac{1}{2}\left[\frac{\alpha a \xi}{V}(N-\bar{N})-\frac{c}{n_{r} L} \varphi\right]+F_{\theta_{p}}(t) \\
\frac{d N}{d t} & =-\sum_{p} A_{p} S_{p}-\frac{N}{\tau_{S}}+\frac{I}{e}+F_{N}(t)
\end{aligned}
$$

where $G_{p}$ is the gain of mode $p$ whose wavelength is $\lambda_{p}, G_{\text {tho }}$ is the threshold gain of the solitary laser and $U_{p}$ is a function counting contribution of OFB to the instantaneous photon number $S_{p}(t)$ of mode $p$. These parameters are defined as,

$$
\begin{aligned}
G_{p} & =A_{p}-B_{p} S_{p}-\sum_{q \neq p}\left(D_{p(q)}+H_{p(q)}\right) S_{q} \\
G_{\text {tho }} & =\frac{c}{n_{r}}\left[k+\frac{1}{2 L} \ln \frac{1}{R_{f} R_{b}}\right] \\
U_{p} & =1+\left(1-R_{f}\right) \sqrt{\frac{\eta \Gamma}{R_{f}}} \exp \left(-j \omega_{p} \tau\right) \sqrt{\frac{S_{p}(t-\tau)}{S_{p}(t)}} \exp \left(j\left\{\theta_{p}(t-\tau)-\theta_{p}(t)\right\}\right) \\
& =\left|U_{p}\right| \exp (-j \varphi) \\
\varphi & =-\tan ^{-1} \frac{\operatorname{Im} U_{p}}{\operatorname{Re} U_{p}}+m \pi \\
I=I_{D} & +I_{M} \cos \left(2 \pi f_{M} t\right)
\end{aligned}
$$

The noise sources $F_{S p}(t), F_{S \theta}(t)$ and $F_{N}(t)$ are added to the rate equations to describe the intrinsic fluctuations in the lasing field due to inclusion of the incoherent spontaneous emission into the stimulated emission.

Here, $a$ is the differential gain coefficient, $\xi$ is the field confinement factor, $V$ is the volume of the active region, $\lambda_{0}$ is the peak wavelength, $\delta \lambda$ is the half-width of spontaneous emission, $\alpha$ is the linewidth enhancement factor, $\bar{N}$ is the time average value of $N(t), \tau_{s}$ is the electron lifetime, $I$ is the injection current, $e$ is the electron charge, $A_{p}$ is the linear gain, $B_{p}$ is the coefficient of self-suppression, $D_{p(q)}$ and $H_{p(q)}$ are the coefficients of the symmetric gain saturation and the asymmetric gain saturation, respectively, $k$ is the internal loss in the laser cavity, $\eta$ is the coupling coefficient into the active region, $\Gamma$ is the optical feedback ratio to the laser cavity, $\omega_{p}=2 \pi c / \lambda_{p}$ is the angular frequency of mode $p, \omega_{p} \tau$ is the phase delay of the field in each round-trip time, $I_{D}$ is the bias current, $I_{M}$ is the modulation current and $f_{M}$ is the frequency of sinusoidal modulation. 


\section{REDUCTION OF OFB NOISE}

The simulated spectra of RIN profiles of the OFB noise and suppressed noise by superposition of $\mathrm{HF}$ current are shown in Fig. 2. Quantum noise spectra are also shown for comparison. The noise is increased more than 20dB by the OFB in lower frequency region due to unstable mode hopping between two lasing modes [3]. However, the RIN is well suppressed by introduction of the superposition of HF current. Line spectrum in the figure indicates modulation of the photon number with the HF current and its higher harmonics.

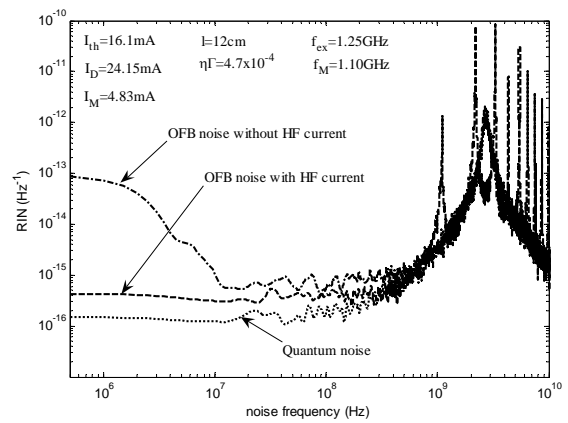

Fig. 2. The simulated spectra of RIN profiles of the OFB noise and suppressed noise by superposition of HF current.

Suppression of the OFB noise by the superposition of HF current is not always effective. Dependency of the modulation frequency $f_{M}$ of the HF current for noise suppression is shown in Fig. 3. The noise is reduced in wide range of the modulation frequency $f_{M}$. However, the RIN raises up when modulation frequency $f_{M}$ of the superposed current coincides with a rational number of the round trip time period $f_{e x}$.

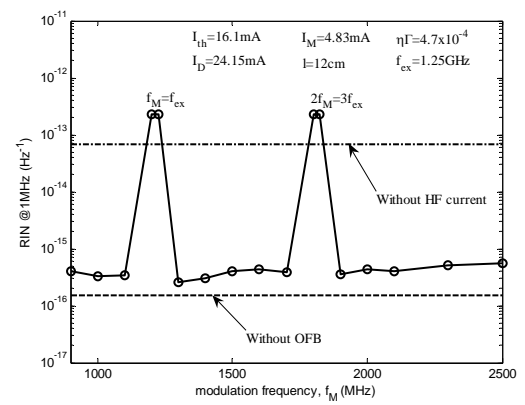

Fig. 3. Calculated data showing dependence of the RIN on modulation frequency of the superposed HF current.

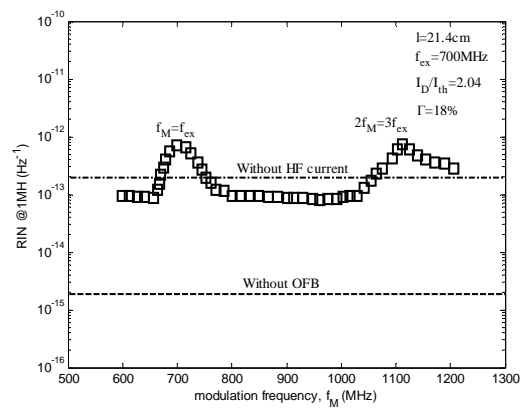

Fig. 4. Experimental data showing dependence of the RIN on modulation frequency of the superposed HF current [2].

The experimental data in Fig. 4 shows the same evidence that the OFB noise raises up when $f_{M}$ and $f_{e x}$ are in rational relations, that is, when $n f_{M}=m f_{e x}$.

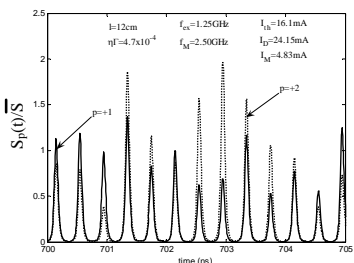

(a)

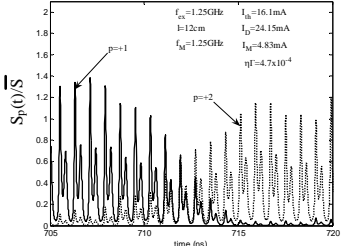

(b)
Fig. 5. Temporal variations of lasing modes. (a) is with condition $n f_{M} \neq m f_{e x}$ and (b) is with condition $2 f_{M}=3 f_{e x}$.

Condition to suppress the OFB noise is that, variations of the electron number and the photon number are large enough and be in same phase. In this case, temporal variations of the gain $G_{p}$ and the contribution of the OFB $C_{p}=\left(c / n_{r} L\right) \ln \left|U_{p}\right|$ are not synchronized, and the phase difference between feedbacked light and emitting light has not maintain any fixed relation. Then variations of the electron number $N$ and the gain coefficient $G_{p}$ are not disturbed by the OFB, resulting in sufficient variation of the photon number $S_{p}$. Thus the laser reveals stable multimode operation, as shown in Fig. 5(a), with which the OFB noise is well suppressed.

In the case of $n f_{M}=m f_{e x}$, variation of the electron number and that of the photon number have $90^{\circ}$ phase difference. We find that the variations of $G_{p}$ and $C_{p}$ are synchronized with $f_{M}$ having $180^{\circ}$ phase difference. Then the phase difference between feedbacked light and emitting light is locked with rational frequency of $f_{M}$ and works to reduce variation of $G_{p}+C_{p}$. As a result, the modulation of the photon number is reduced as found from Eqn. (1) and the OFB noise is increased with mode hopping remained as shown in Fig. 5(b).

\section{CONCLUSIONS}

A simulation model giving generation of the OFB noise and its suppression by the superposition of $\mathrm{HF}$ current in semiconductor lasers is presented in this paper. The superposition of HF current works to modulate both the electron number $N$ and the photon number $S_{p}$, which works to stop mode hopping resulting in suppression of the OFB noise. However, the noise suppression effect does not work under the condition $n f_{M}=m f_{e x}$ for modulations of the electron number $N$ and the photon number $S_{p}$ are suppressed by the phase locking effect with undesirable phase relation.

\section{REFERENCES}

[1] K. Petermann, Laser Diode Modulation and Noise, Boston, MA: Kluwer, 1991.

[2] M. Yamada, S. Yamamura and T. Okamoto, "Characterization of the feedback induced noise in semiconductor lasers under superposition of high frequency current”, IEICE Trans. Electron., vol. E84-C, no. 10, Oct. 2001.

[3] S.M.S. Imran, M. Yamada and Y. Kuwamura, "A theoretical analysis of the optical feedback noise based on multimode model of semiconductor lasers”, IEEE J. Quantum Electron., vol. 48, no. 4, pp. 521-527, April 2012. 